\title{
Traveling and Standing Waves in Binary-Fluid Convection in Finite Geometries
}

\author{
M. C. Cross \\ California Institute of Technology, Pasadena, California 91125 \\ (Received 30 June 1986)
}

\begin{abstract}
The effect of finite geometry on the competition between traveling waves and standing waves in systems with a Hopf bifurcation to a state with spatial structure is considered in the linear and weakly nonlinear regimes. The spatial structure observed by Kolodner et al. in binary-fluid convection is explained in terms of the reflection of the linear traveling waves. The reflection coefficient is calculated, and is found to go to zero as the frequency of the waves becomes small. The pattern expected in a saturated nonlinear state is discussed.
\end{abstract}

PACS numbers: 47.25.-c

Convection in binary-fluid mixtures provides a rich system for the study of nonequilibrium phenomena. For example, the first instability to a state with spatial structure- the convection rolls-varies from a stationary bifurcation to an oscillatory (Hopf) bifurcation as a parameter of the fluid is changed. ${ }^{1}$ Questions in pattern formation in a dynamical context may be investigated in a well-controlled fluid system. In addition, there is the possibility of interesting dynamical behavior near the stationary-oscillatory crossover point., ${ }^{2,3}$ (I will follow the general usage and call this point the codimension-two point.) There has been a flurry of experiments ${ }^{4}$ to investigate these questions. However, one of the simplest theoretical questions has not yet been addressed properly - the spatial structure of the periodic solutions in a finite lateral geometry. In a laterally infinite system standing waves are thought to be unstable to traveling waves. There is, however, a considerable literature ${ }^{2,5}$ on the properties of standing-wave solutions, at first sight the natural solutions in a finite geometry. The present work addresses the question of the competition between traveling waves and standing waves in finite geometries in both the linear and weakly nonlinear situations.

I discuss the competition in terms of a slightly simplified model system, commonly used in the literature. This gives us a rather complete understanding of the recent experiments of Kolodner et al. ${ }^{6}$ : In particular I find that the length scale of variation of the envelope of the traveling waves observed there should scale with the length of the system, and gives us information on the reflection of the waves at the lateral boundaries. I calculate the reflection coefficient numerically for the model system, and obtain an analytic expression for small oscillation frequencies. The experiment apparently may be understood in a linear theory. In addition I discuss the use of envelope equations ${ }^{7}-$ a very successful approach to stationary convection near threshold-in the weakly nonlinear region. Only where the propagation speed (group velocity) is small do the envelope equations yield a complete description. The steady solutions in this limit are briefly discussed.
In some beautiful experiments in alcohol-water mixtures Kolodner et al. ${ }^{6}$ have studied the oscillatory convection state over the long transient period when the amplitude is growing exponentially, indicating that nonlinear effects are playing a minor role. ${ }^{8}$ They observe a pattern of straight rolls perpendicular to the long dimension of the rectangular cell. In the center the pattern is a superposition of roughly equal amplitudes of leftward and rightward traveling waves. Towards the ends one or other of the waves dominates. The authors parametrize the disturbance in terms of an amplitude, which I will call $\chi(x, t)$ :

$$
\begin{aligned}
\chi(x, t)=\chi_{0} e^{\gamma t}\left[e^{\beta x} \cos (k x\right. & -\Omega t) \\
& \left.-e^{-\beta x} \cos (k x+\Omega t)\right] .
\end{aligned}
$$

The length scale $\beta^{-1}$ is surprisingly long-about $12.5 d$, with $d$ the cell depth, for separation coefficient $\Psi=-0.57$.

We can understand this form in terms of leftward and rightward traveling waves with amplitudes $\chi_{R}$ and $\chi_{L}$ : $\chi=\chi_{R}+\chi_{L}+$ c.c. with

$$
\chi_{R, L}=a_{R, L} e^{\lambda( \pm x-s t)} e^{\bar{\varepsilon} t / \tau_{0}} e^{i( \pm k x-\Omega T)},
$$

connected by reflecting boundary conditions at the ends $x= \pm \frac{1}{2} l$, e.g.,

$$
\chi_{L}\left(\frac{1}{2} l\right)=r \chi_{R}\left(\frac{1}{2} l\right)
$$

where $r=|r| \exp \left(i \phi_{r}\right)$ is the reflection coefficient. Equation (2) is comprised of the basic traveling waves with wave number $k$ and frequency $\Omega$ modulated by slowly varying amplitudes $A_{R, L}(x, t)$.

Here $s$ is the group velocity $d \Omega(k) / d k$, so that in a comoving frame the growth rate is the characteristic $\bar{\varepsilon}^{-1} \tau_{0}$ with $\bar{\varepsilon}$ approximately ${ }^{9}$ equal to $\varepsilon$, given in terms of the Rayleigh number $R$ and its value $R_{\mathrm{co}}$ at the oscillatory threshold by $\varepsilon=\left(R-R_{\text {co }}\right) / R_{\text {co }}$. The exponential dependence on the comoving coordinates $x \pm s t$ is confirmed by the linear analysis of the envelope equations (see below). Equations (2) and (3) reproduce the form 
observed with the identifications $\gamma=\bar{\varepsilon} \tau_{0}^{-1}-\lambda s$ for the temporal growth rate and $\beta=\lambda$ for the spatial envelope with $\lambda^{-1}=-l / \ln (|r|)$. Notice that the length scale is predicted to scale simply with the length of the system, and gives direct information on the reflection amplitude. The experimental value of $\lambda$ at $\Psi=-0.57$ yields $|r|$ $=0.31$. Note also that there is a shift in the onset $(\gamma=0)$, given by $\bar{\varepsilon}=\lambda s \tau_{0}$, that is linear in $l^{-1}$ and dependent on $r$ : The growth of the wave during a passage over the length of the cell must overcome the reduction in amplitude due to reflection. Perfect reflection would give standing waves in the linear regime.

I have calculated the reflection coefficient expected at a rigid side wall for the simplified model which uses boundary conditions at the upper and lower plates corresponding to free fluid slip and plates that are permeable to the finite-wave-number concentration perturbations. ${ }^{10}$ The equations for the velocity field $u$ (vertical component $w$ ), pressure field $P$, temperature perturbation $\theta$, and impurity concentration perturbation $c$ in the usual "Boussinesq" approximation" are

$$
\begin{aligned}
& \dot{\mathbf{u}}+(\mathbf{u} \cdot \nabla) \mathbf{u}=-\nabla P+\sigma(\theta-c) \hat{\mathbf{z}}+\sigma \nabla^{2} \mathbf{u}, \\
& \dot{\theta}+(\mathbf{u} \cdot \nabla) \theta=R w+\nabla^{2} \theta, \\
& \dot{c}+(\mathbf{u} \cdot \nabla) c=-R \Psi w+L \nabla^{2} c+\Psi L \nabla^{2} \theta, \\
& \nabla \cdot \mathbf{u}=0 .
\end{aligned}
$$

The equations have been rendered dimensionless in the usual way (lengths scaled with the depth, times with the vertical thermal diffusion time), and the properties of the system are parametrized by the Prandtl number $\sigma$, the Lewis number $L$, and the separation coefficient $\Psi$, together with the Rayleigh number $R$. The Lewis number, the ratio of impurity diffusivity to thermal diffusivity, is typically very small, and we may drop terms proportional to $L$ in Eq. (6). In the linear regime, and for an oscillatory solution $\exp [-i \Omega t], c$ is then algebraically related to $w$, the vertical velocity. We are interested in the behavior near the threshold $R_{\mathrm{co}}=\left(27 \pi^{4} / 4\right)(1+\sigma)(1$ $+\sigma+\sigma \Psi)^{-1}$ to the oscillatory states with frequency $\Omega^{2}=\left(9 \pi^{4} / 4\right)(-\Psi \sigma)(1+\sigma+\sigma \Psi)^{-1}$ giving a disturbance in the bulk of the form

$$
(w, \theta, c)_{i}=\left(w_{0}, \theta_{0}, c_{0}\right) \sin \pi z \exp [i(k x-\Omega t)],
$$

with $k=\pi / \sqrt{2}$ and $w_{0}, \theta_{0}, c_{0}$ numbers given by solving for the eigenvectors of Eqs. (4)-(7)

To calculate the reflection coefficient, consider the wave equation (8) incident from left on a rigid side wall. In a boundary layer region of thickness $\sim 1$, exponentially decaying solutions are excited so as to satisfy the fluid boundary conditions $w=0$ and $u=0$ and the thermal conditions (e.g., $\theta=0$ for good conductors, $\partial_{x} \theta=0$ for insulators). ${ }^{12}$ In addition a reflected wave of amplitude $r$ is excited. For the free-slip permeable boundary conditions the equations are separable, and we need only solve algebraic equations for the exponential decay rates of solutions varying as $\sin \pi z$. Values of $|r|$ predicted for various values of $\sigma$ and $\Psi$ and for side walls of good and bad thermal conductors are shown in Table I.

It is apparent that as $-\Psi$ approaches zero, and the frequency of the oscillations becomes small, the reflection coefficient also becomes small. We can analyze this limit analytically in terms of envelope equations for the slow amplitudes $A_{R}(x, t), A_{L}(x, t)$ which then take the form

$$
\begin{array}{r}
\dot{A}_{R}=\tau_{0}^{-1}\left(\varepsilon A_{R}+\xi_{0}^{2} \partial_{x}^{2} A_{R}\right)-s \partial_{x} A_{R} \\
-g_{1}\left|A_{R}\right|^{2} A_{R}-g_{2}\left|A_{L}\right|^{2} A_{R}, \\
\dot{A}_{L}=\tau_{0}^{-1}\left(\varepsilon A_{L}+\xi_{0}^{2} \partial_{x}^{2} A_{L}\right)+s \partial_{x} A_{L} \\
-g_{1}\left|A_{L}\right|^{2} A_{L}-g_{2}\left|A_{R}\right|^{2} A_{L} .
\end{array}
$$

Note that, more generally, ${ }^{7,13}$ the coefficients of each of the terms would be complex and $s$, the group velocity, would be $\sim 1$. The solutions of Eqs. (4)-(7) in finite geometries then vary on an $\sim 1$ length scale that is inconsistent with the slow variation assumed: Equations (9) do not yield a complete solution in this general case. Also note that a simple envelope equation for a spatially varying standing wave ${ }^{2}$ (i.e., a single equation for $A_{R}+A_{L}$ ) cannot be written down because of the propagation terms. However, on approach to codimension-two point where $\Omega$ goes to zero as $(-\Psi)^{1 / 2}$, the group velocity $s$ becomes small, and the solutions vary on a slow length scale. In this limit also the imaginary parts of the coefficients become small. Equations (9) then allow us to study oscillatory convection in finite boxes in both the linear and nonlinear regimes. ${ }^{14}$

The envelope equations must also be supplemented by boundary conditions that reflect the consequences on $A_{R}$ and $A_{L}$ of the boundary-layer region. For $L \rightarrow 0$ and small $\Omega$ these take the form (at $x= \pm \frac{1}{2} l$ )

$$
\begin{aligned}
& A_{R}-\alpha_{ \pm} A_{R}^{\prime}-\beta_{ \pm} A_{L}^{\prime}=0, \\
& A_{L}-\alpha_{ \pm}^{*} A_{L}^{\prime}-\beta_{ \pm}^{*} A_{R}^{\prime}=0,
\end{aligned}
$$

TABLE I. Values of the magnitude of the reflection coefficient $|r|$ at rigid side walls of good or bad thermal conductivity (relative to the fluid). The first two values are those used in Ref. 6; the remainder show the general trends. For $(-\Psi)^{-1} \gg \sigma \gg 1$ there is very little dependence on the Prandtl number.

\begin{tabular}{rccc}
\hline \hline & \multicolumn{2}{c}{ Reflection coefficient $|r|$} \\
\multicolumn{1}{c}{$\sigma$} & $\Psi$ & Good conductor & Bad conductor \\
\hline 14.9 & -0.58 & 0.31 & 0.26 \\
9.8 & -0.29 & 0.18 & 0.26 \\
10.0 & -0.80 & 0.458 & 0.245 \\
10.0 & -0.60 & 0.327 & 0.272 \\
10.0 & -0.40 & 0.230 & 0.274 \\
10.0 & -0.20 & 0.144 & 0.246 \\
10.0 & $-10^{-4}$ & $3.0 \times 10^{-3}$ & $9.9 \times 10^{-3}$ \\
\hline \hline
\end{tabular}


with $\alpha_{+}=-\alpha_{-}^{*}=\alpha$ and $\beta_{+}=-\beta_{-}^{*}=\beta$ with $\alpha$ and $\beta$ identical to the coefficients already calculated for stationary convection in pure fluids. ${ }^{15}$ The primes in Eq. (10) denote $x$ derivatives.

The reflection coefficient of linear waves is now easily calculated to give $r \simeq-\beta^{*} s \tau_{0} / \xi_{0}^{2}$. Using the values calculated from the linearized fluid equations, i.e., $\tau_{0}$ $=4\left(1+\sigma^{-1}\right) /\left(3 \pi^{2}\right)$ and $\xi_{0}^{2}=8 /\left(3 \pi^{2}\right)$ and $s=\left[2 \pi^{2}\right.$ $\times \sigma(-\Psi) /(1+\sigma+\sigma \Psi)]^{1 / 2}$, and the values of $\beta$ from Brand, Hohenberg, and Steinberg, ${ }^{16}$ i.e., $|\beta|=0.431$ for poor conductors and $|\beta|=0.130$ for good conductors, we can calculate the reflection coefficient. For $\Psi^{-1} \gg \sigma \gg 1$ the dependence on the Prandtl number disappears, and $|r|=0.96(-\Psi)^{1 / 2}$ for poor conductors and $0.29(\Psi)^{1 / 2}$ for good conductors. Note that $|r|$ varies proportional to the frequency $\Omega$ as $\Omega \rightarrow 0$, and we expect this behavior to be evident with the realistic boundary conditions, at least for not too small frequencies.

I have analyzed the situation of weakly nonlinear waves in the low-frequency limit, assuming $g_{1}>0$ (i.e., the amplitude of the traveling waves saturates) and $g_{2} / g_{1}>2$ (standing waves in the bulk are unstable to traveling waves $\left.{ }^{13}\right)$. It is convenient to rescale the equations to eliminate redundant constants $\left(X=\varepsilon^{1 / 2} x / \xi_{0}\right.$; $T=\varepsilon^{1} t / \tau_{0} ; \quad \bar{A}_{R, L}=\varepsilon^{-1 / 2} g_{1}^{1 / 2} A_{R, L} ; \quad \bar{s}=s \varepsilon^{-1 / 2} \tau_{0} / \xi_{0} ; \quad$ and $\bar{g}_{2}=g_{2} / g_{1}$ ). The boundary conditions are as Eq. (10) with $A \rightarrow \bar{A}, \alpha, \beta \rightarrow \bar{\alpha}, \bar{\beta}$, and $\bar{\alpha}=\varepsilon^{1 / 2} \alpha / \xi_{0}, \quad \bar{\beta}=\varepsilon^{1 / 2} \beta / \xi_{0}$ both small numbers. This situation may apply to the slowly moving waves observed at larger amplitudes in the bulk fluid convection ("overturning convection"6), to binary fluid convection in a porous medium near the codimension-two point, ${ }^{16}$ and qualitatively to the general situation and other systems.

I have investigated the stationary solutions of Eqs. (9), corresponding to simply periodic solutions of the original equations, using a finite-difference Newton-Raphson scheme. (Work on the direct time evolution is in progress, and will be reported elsewhere.) Boundary conditions Eq. (10) are used at $x=-\frac{1}{2} l$, and these, or conditions matching to saturated solutions in a semifinite system, at $x=+\frac{1}{2} l$. I take $\bar{\alpha}, \bar{\beta}$ to be real for simplicity, so that $A_{R, L}$ may be taken real. Here I discuss the main qualitative conclusions.

From work on propagating fronts in the Fisher equation ${ }^{17}$ the value $\bar{s}=2$ might be anticipated to be a critical value, and different behaviors are indeed apparent on either side (although the exact boundary value may also depend on $\bar{\alpha}, \bar{\beta})$. A typical solution in a large box for $\bar{s}<2$ is shown at the top of Fig. 1. Notice the predominance of one component, here the one moving to positive $x$, with a small reflected amplitude of the second component at $x=\frac{1}{2} l$ that is suppressed in the bulk by the nonlinear terms. Another class of solutions exists, with $A_{R}$ large for $x>0$ and $A_{L}$ large for $x<0$ [cf. the linear solution Eq. (1)]. In either case traveling waves are apparent over most of the length, except for short healing
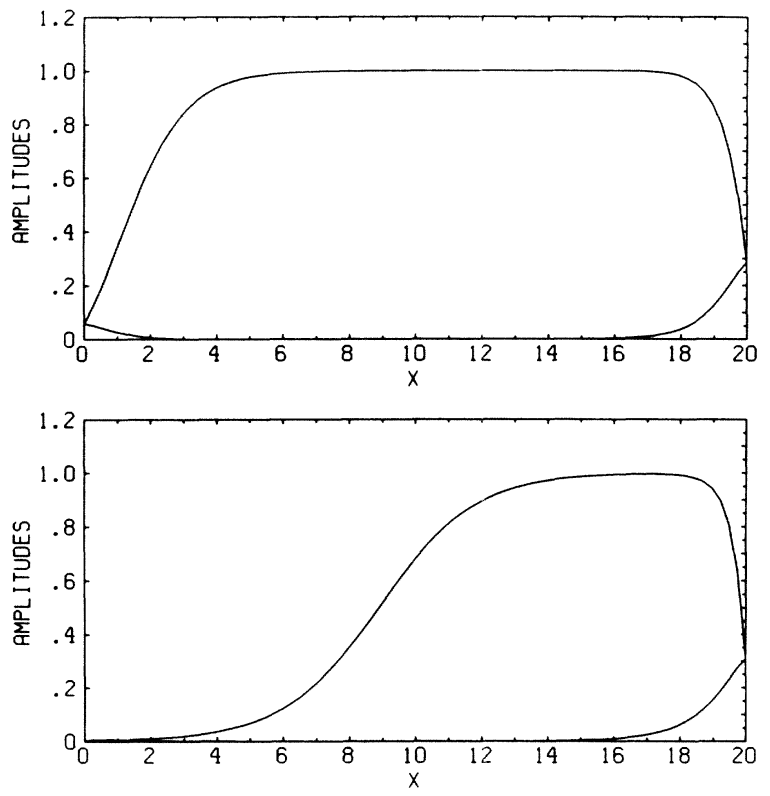

FIG. 1. Comparison of the amplitudes $\bar{A}_{R}(X)$ and $\bar{A}_{L}(X)$ for $\bar{s}<2$ (upper figure) and $\bar{s}>2$ (lower figure). In both cases the rightward moving wave $\bar{A}_{R}$ is dominant. The parameters used are $l=20$ and $\bar{g}=4$, with $\bar{s}=1.0, \bar{\alpha}=\bar{\beta}=0.30$ in the upper figure and $\bar{s}=2.2, \bar{\alpha}=\bar{\beta}=0.18$ in the lower figure.

regions at the ends, or at the junction of the two sets. The amplitude saturates in the bulk, and the values $\bar{A}_{R, L}$ at the boundaries and the lengths over which the amplitudes are suppressed are independent of the length for long systems. A saturated nonlinear state is a solution in the semi-infinite system.

For $\bar{s} \gtrsim 2$ the situation is quite different. With boundary conditions at $x=\frac{1}{2} l$ matching to the semi-infinite system no steady-state solution is found: The disturbance apparently propagates away to large distances. A steady-state solution exists in a large box, but now with the reflected $A_{L}$ wave necessary to excite, on a second reflection at $x=-\frac{1}{2} l$, the $A_{R}$ wave. The magnitude of $A_{R, L}$ at the growth boundary $x=-\frac{1}{2} l$ consequently decreases rapidly, presumably exponentially, as the length grows, and the distance over which $A_{R}$ is small scales with the size of the system. The convection is suppressed over a large fraction of the cell. The growth of $A_{R}$ away from $x=-\frac{1}{2} l$ is as $\exp (x / \bar{s})$ and becomes slow for large $\bar{s}$. (By contrast, the decay rate at $x=+\frac{1}{2} l$ becomes fast as $\bar{s}$, and cannot be treated with the envelope equations for large $\bar{s}$.) Since the amplitude grows slowly from an exponentially small value near the $x=-\frac{1}{2} l$ boundary, by analogy with our experience of the envelope equation for stationary bifurcations, ${ }^{15,18}$ I would expect a rather precise wave-number selection in this dynamical situation. This requires further investigation.

The author thanks A. Karma for useful discussions. This work was supported under Division of Materials 
Research Grant No. DMR-8412543 by the National Science Foundation.

${ }^{1}$ The theoretical assertion that this is true in bulk fluid mixtures is based on approximate calculations, or the simple model system used by D. T. J. Hurle and E. Jakeman, J. Fluid Mech. 47, 667 (1971); R. S. Schechter, M. G. Velarde, and J. K. Platten, in Advances in Chemical Physics, edited by I. Prigogine and S. A. Rice (Wiley, New York, 1974), Vol. 26, p. 265; D. Gutkowicz-Krusin, M. A. Collins, and J. Ross, Phys. Fluids 22, 145 (1979); and H. R. Brand, P. C. Hohenberg, and V. Steinberg, Phys. Rev. A 30, 2548 (1984).

${ }^{2}$ Brand, Hohenberg, and Steinberg, Ref. 1.

${ }^{3}$ B. J. A. Zielinska, D. Mukamel, V. Steinberg, and S. Fishman, Phys. Rev. A 30, 702 (1985).

${ }^{4}$ I. Rehberg and G. Ahlers, Phys. Rev. Lett. 55, 500 (1985); H. Gao and R. P. Behringer, Bull. Am. Phys. Soc. 30, 1719 (1985); R. W. Walden, P. Kolodner, A. Passner, and C. M. Surko, Phys. Rev. Lett. 55, 496 (1985).

${ }^{5}$ L. N. Da Costa, E. Knobloch, and N. O. Weiss, J. Fluid Mech. 25, 109 (1981); D. R. Moore, J. Toomre, E. Knobloch, and N. O. Weiss, Nature (London) 303, 663 (1983).

6P. Kolodner, A. Passner, C. M. Surko, and R. W. Walden, Phys. Rev. Lett. 56, 2621 (1986).

${ }^{7}$ A. C. Newell, in Synergetics, edited by H. Haken (Springer, New York, 1977), Vol. 2.
${ }^{8}$ For the model system described below the usual lowestorder nonlinear saturating term is identically zero for traveling waves [C. S. Bretherton and E. A. Spiegel, Phys. Lett. 96A, 152 (1983)]. Furthermore, a mode truncation scheme for traveling waves in this model shows no saturation at small amplitudes near threshold IM. C. Cross, Phys. Lett. A (to be published)] in agreement with experiment.

${ }^{9}$ There is a quadratic shift than can be derived from Eq. (9): $\bar{\varepsilon}=\varepsilon+\xi_{0}^{2} \lambda^{2}$.

${ }^{10}$ At first sight, permeable boundary conditions seem entirely unphysical. However, outside a boundary layer of thickness $(L / \Omega)^{1 / 2}$ effective permeable conditions exist.

${ }^{11}$ Hurle and Jakeman, Ref. 1.

${ }^{12}$ For $L \rightarrow 0$ the concentration boundary condition will again be satisfied over a very small lateral boundary layer and does not affect the analysis elsewhere.

${ }^{13}$ P. Coullet, S. Fauve, and E. Tirapegui, J. Phys. (Paris), Lett. 46, 787 (1985).

${ }^{14} \mathrm{~A}$ single equation of this form, e.g., for $A_{R}$, has been considered by S. Zaleski, P. Tabeling, and P. Lallemand, Phys. Rev. A 32, 655 (1985), in the context of Taylor Couette flow.

${ }^{15}$ M. C. Cross, P. G. Daniels, P. C. Hohenberg, and E. D. Siggia, J. Fluid Mech. 127, 155 (1983).

${ }^{16}$ H. R. Brand, P. C. Hohenberg, and V. Steinberg, Phys. Rev. A 27, 591 (1983).

${ }^{17}$ E. Ben-Jacob, H. Brand, G. Dee, L. Kramer, and J. S. Langer, Physica (Amsterdam) 14D, 348 (1985).

${ }^{18}$ L. Kramer, E. Ben-Jacobs, H. Brand, and M. C. Cross, Phys. Rev. Lett. 49, 1891 (1981). 